\title{
Pseudo-CT Generation for External Radiotherapy Planning from Magnetic Resonance Imaging Data on Brain Regions
}

\author{
Niloofar Yousefi Moteghaed ${ }^{1}$, Ali Yaghobi Joybari ${ }^{2}$ and Ahmad Mostaar ${ }^{1,3,{ }^{*}}$ \\ ${ }^{1}$ Department of Medical Physics and Biomedical Engineering, School of Medicine, Shahid Beheshti University of Medical Sciences, Tehran, Iran \\ ${ }^{2}$ Department of Radiation Oncology, School of Medicine, Shahid Beheshti University of Medical Sciences, Tehran, Iran \\ ${ }^{3}$ Radiation Biology Research Center, Iran University of Medical Sciences, Tehran, Iran \\ "Corresponding author: Department of Medical Physics and Biomedical Engineering, School of Medicine, Shahid Beheshti University of Medical Sciences, Tehran, Iran. Tel: \\ +98-2123872566, Fax: +98-2122439941, Email: mostaar@sbmu.ac.ir
}

Received 2018 July 31; Revised 2019 April 26; Accepted 2019 May 11.

\begin{abstract}
Background: Nowadays, medical imaging has an important role in radiotherapy and treatment planning process. Despite the increasing usage of the magnetic resonance imaging (MRI) in the external radiotherapy (RT) design process, computed tomography (CT) remains a basic imaging modality in radiotherapy because of its relation with electron density value. In conventional external radiotherapy, MRI is used in functional tissue structures with registration on the CT image, which causes systematic errors during the registration of MRI and CT images.

Objectives: The main purpose of this paper was to investigate the possibility of removing the CT simulator and replacing it with pseudo-CT for the electron density calculation in radiotherapy treatment planning.

Materials and Methods: The pseudo-CT images were generated for 10 randomly chosen patients with brain disease. The data consisted of image voxels chosen within the segmented area of the brain in both MRI and CT images. The relationship between electron density information in CT images and MRI intensity value was derived from simple polynomial models.

Results: It was found that the MRI intensity value is related to the Hounsfield unit (HU) value within different parts including the skull bone, sinus, and brain in CT images. The mean errors of the conversion model were $0.7479,0.334$ and $-0.043 \mathrm{HU}$ in the brain, skull, and air regions respectively, with the simple polynomial model in the upper slice.

Conclusion: The proposed method generated pseudo-CT images from different segmented parts of the brain of MRI series. The application requires segmentation of the cortical bones in MR images. The result indicated that finding a complex model does not necessarily lead to achieving a better result.
\end{abstract}

Keywords: Magnetic Resonance Imaging, Computed Tomography, Pseudo-CT, Radiotherapy Treatment Planning

\section{Background}

Nowadays, computed tomography (CT) is critical for the technical aspects of three dimensional (3D) conformal radiotherapy and image-guided external radiation therapy dose planning. CT images provide a relationship between tissue electron density and voxel values to set up accurate dose calculations, which depend on the attenuation properties of the tissue and generation of digitally reconstructed radiographs (DRRs) (1). In recent years, interest has been developing in replacing CT scan images with MRI images in the treatment planning process. This is due to the fact that MRI provides a high soft tissue contrast that could improve the determination of target tissue and the accuracy of risk volume delineation in organ at risk (OR). The advantages of planning directly on MRI scans include numbers of functional imaging options, no ionizing radi- ation for the patient, and reduction in costs (1-4).

Higher contrast in the soft tissue can lead to better separation of tumor tissue from the organ at risk and determine the exact size of the tumor area (5). Furthermore, MRI can be used before, during, and after treatment for patient follow-up without being concerned about patients receiving ionizing radiation (6). MRI is going to be used as a tool for image-guided radiotherapy (IGRT), where MRICobalt and MRI-Linac are being developed and even these machines have been used in some limited radiation therapy centers (7). In the treatment planning dose calculation in MR-Linac, the entire patient volume is assumed as water equivalent electron density. Furthermore, assuming a homogeneous density compared with planning heterogeneously can lead to dose discrepancies greater than $2 \%$. So, the tissues in the MR image were classified into different 
classes such as soft tissue and bone (and in some cases air) by manual contouring from T1-weighted MR images and every class was assigned an electron density (8).

Nowadays, based on fusion of MRI images with CT scan slices in soft tissue structures, both images can be taken advantage of simultaneously (9). Nevertheless, in addition to increased extra costs and scan time using multiple modalities, fusion techniques have limitations (10). In MRIonly based systems in which the CT modality is eliminated completely, in addition to solving the extra cost, time and limitation issues, brain segmentation could be performed more accurately and more comfortably (11).

Alongside all these advantages, geometric distortions due to non-uniformities of the magnetic field, gradient nonlinearity, and patient-induced susceptibility are some limitations that must be properly investigated in an MRIonly radiation planning to produce accurate treatment planning and dose calculation. (12-14). Also, a significant problem in MRI-alone radiation planning systems, however, is that scans cannot be calibrated to electron density value due to different imaging protocols. There are different methods to provide an electron density map, one of which is rigid registration of MRI images on CT images. Unfortunately, this method will be so difficult when the patient's position is slightly different between MRI and CT imaging $(2,14,15)$. Several researchers have investigated the possibility of removing CT modality from radiation therapy planning and implementing an MR-only simulation system (16-19). Different methods have been developed to estimate the electron density information from MRI for external radiation therapy in recent years. Bulk density assignment involves applying an area of interest within the MRI to single homogeneous density values for manual contouring. The main benefit of the method is its manageability, though the calculation results may not be as accurate as CT for constructing reliable DRRs (14, 15, 2024). Atlas-based registration methods involve registering target MRI to a single CT to generate a substitute CT, which can be a simple approach, unlike accurately mapping complex anatomy. Atlas-based approaches are currently the only fully automated methods for generating pseudo-CT images by converting a single standard MRI sequence to $\mathrm{CT}$, and are more robust to intensity differences between images. The main disadvantage is that the registration algorithms used may be unable to deform atlas images to match anatomical properties which are missing from an atlas-training set (25).

A semi-automated segmentation has been proposed through a deformable registration of a selected atlas (26). A conjugated electron-density mapping atlas and whole MRI atlas based on the manually delineated MRI scans have been generated by Dowling et al. (16). An optimization ap- proach based on the robust block-matching has been proposed which utilizes a half-way space definition to maintain inverse-consistency (27). Also, a half space transform and its inverse have been optimized simultaneously by a robust symmetric registration algorithm (28). Synthesizing CTs from MR images has been done using an iterative multi-atlas approach due to morphological similarity of the mapped atlases to the target (29). To learn the intensity mapping with atlas-based approaches, most regression methods rely on a training set of co-registered CT-MR scans. Other studies have investigated the use of Gaussian Mixture Regression and Random Forest Regression for creating a pseudo-CT image from dual ultra-short echo time (dUTE) and m-Dixon MRI images $(30,31)$. In addition to the two methods mentioned, another way in creating pseudoCT images is voxel-based methods that are embedded in two groups based on the use of functional MRI sequences: the standard sequences and the ultrashort echo time sequences (UTE). In the study (32), pseudo-CTs were generated using a voxel-based, weighted summation method using ultrashort echo time phase images from a weighted combination of water fat maps and unwrapped UTE phase maps. CT image HUs values and T1/T2 weighted MRI intensity values were utilized to generate a model conversion technique from MR intensity by applying a second-order polynomial model (33). In (34), a voxel-wise tissue classification was applied to derive pseudo-CT for optimization ion radiotherapy treatment plans. Pseudo-CT was created with an inversely modulated radiotherapy (IMRT) plan based on an assigning electron density to an anatomic image (35). The purpose of the study (36-38) is to establish pseudo-CT generation using an undersampled ultrashort echo time (UTE)-mDixon pulse sequence by a linear combination of the fuzzy c-means (FCM) membership functions. In the studies (38-40), substitute CT images were derived by Gaussian mixture regression.

\section{Objectives}

The CT scan, despite numerous benefits, does not reveal accurate information about the soft tissue contrast, which is often difficult to distinguish the target tissues of the organ at risk and to determine the volume of the tumor in a typical RT programming. Also, the main challenge is that the brain MRI cannot map the bone very well. The bone signal is lost before being read because of the short $\mathrm{T} 2$ value, and there is no direct correlation between the intensity of the resonance imaging and electron density. CT images include such information, as the intensity in these images is directly related to the electron density of the tissue and provides an accurate geometry of the bone. The main goal of this study was to investigate the possibility 
of removing the CT simulator from the therapeutic process and replacing it with pseudo-CT images and calculating the electron density values for designing patient treatment planning. It is investigated whether there is a relationship between MRI intensity and CT-HU value within different brain regions. The purpose is to find a simple and efficient model that could provide electron density information from the MRI images to generate the synthetics-CT images with an acceptable error rate that could be used in clinical RT treatment planning. Segmentation of the bones was performed by the Fuzzy C-means algorithm in these images.

\section{Materials and Methods}

The MRI was performed by a 1.5 T SIEMENS medical scanner. Repetition time (TR) was $400 \mathrm{~ms}$, Echo time (TE) was 10ms, flip angle was 90, Bandwidth (BW) was $90.9 \mathrm{kHz}$, and matrix size was $512 \times 512$. The brain CT images were taken by a SIEMENS scanner, at $120 \mathrm{kVp}$ with the slice thickness of $2 \mathrm{~mm}$, and $512 \times 512$ in-plane image dimensions.

Ten randomly chosen patients were prescribed external brain RT (five men and five women, age 20 to 71 years, mean age of 45 years). The patient's agreements were obtained after they were informed about the whole procedure of the study.

This project did not have any effect on the treatment planning of these patients. Eight patients were included in the generation of the model, while two others were considered for independent validation. At first, the series of CT images and MRI images were segmented by the FCM clustering algorithm including soft tissue (brain), air and bone. Clustering is an unsupervised learning algorithm whereby the samples are divided into categories whose members are similar to each other that are called clusters. The cluster is, therefore, a set of objects that are similar to each other and are identical to objects in other clusters. Indeed, agents were selected for input samples and then based on their similarity with the samples, the selected cluster could be determined, which was repeated until the agents of clusters did not change (41). For each patient 10,25 and 5 voxels were chosen randomly within the skull bone, brain, and sinus area, respectively. The corresponding MRI intensity values and CT values of different voxels in different areas created the data points for the generation and validation of the models. The skull voxels were chosen uniformly within the segmented bone volume.

The purpose was to assess the overall relationship between MRI intensity and CT-values in different segmented parts of the brain with sufficient accuracy for radiation therapy planning. The electron density values should be determined from the MRI intensity values. We considered the air regions in CT and MRI series as similar. The model fit is illustrated in Figure 1 for the brain and skull region.

We can use simple polynomial models (1st and 2nd order) for these parts.

$$
\begin{aligned}
& C T_{\text {intensity }}=B+A M R_{\text {intensity }} \\
& \text { Brain: } \mathrm{A}=0.01563, \mathrm{~B}=1042 \\
& \text { Skull: } \mathrm{A}=-0.9004, \mathrm{~B}=2381 \\
& C T_{\text {intensity }}=C+B M R_{\text {intensity }}+A M R_{\text {intensity }}^{2} \\
& \qquad \text { Brain: } \mathrm{A}=3.955 \times 10^{-6}, \mathrm{~B}=0.013, \mathrm{C}=1043 \\
& \quad \text { Skull: } \mathrm{A}=0.003709, \mathrm{~B}=-1.915, \mathrm{C}=2429 \\
& \text { Where, } \mathrm{MR} \text { intensity and } \mathrm{A}, \mathrm{B}, \mathrm{C} \text { represent the MR intensity } \\
& \text { value and fitting parameters of the regression model, re- } \\
& \text { spectively. The parameters include complex functions of } \\
& \text { the tissue component fractions as well as T1 and T2 value } \\
& \text { within a voxel of MR image. The order for the polynomial } \\
& \text { fit was chosen based on the mean absolute error between } \\
& \text { pseudo-CT and simulated CT. Figure } 2 \text { displays the whole } \\
& \text { process of the algorithm by a simple flowchart. }
\end{aligned}
$$

\section{Results}

We chose two simple models for different segmented parts of the brain and bone area to be applied on the test MR images. Figure 3 reveals the simple result of the process.

As the figure indicates, the head area is segmented into different regions by the FCM algorithm. Considering the interrelationships between the data extracted from the image series, and applying them on the test MR values to form pseudo-CT images, the desired result could be achieved.

Figure 4 presents the results of applying the 1st polynomial model to different slices of the head. Two simple models for different segmented parts of the brain and skull region were selected for application to the test MR images.

Two models were applied to test the segmented images in order to demonstrate model functionality. The difference between the three segments in the original and pseudo-CT image can be observed in Figure 5.

For further investigation, Figure 6 reveals the histogram chart of the differences between a selected region in the head and skull region of a pseudo-CT image and the original CT scan using the two models presented in the previous section.

The pseudo-CT images could be compared with the original CT images to verify their characteristics. Possibly, the simplest and the most common measurement scale is the measure of voxel-wise mean absolute error and the mean error as below: 

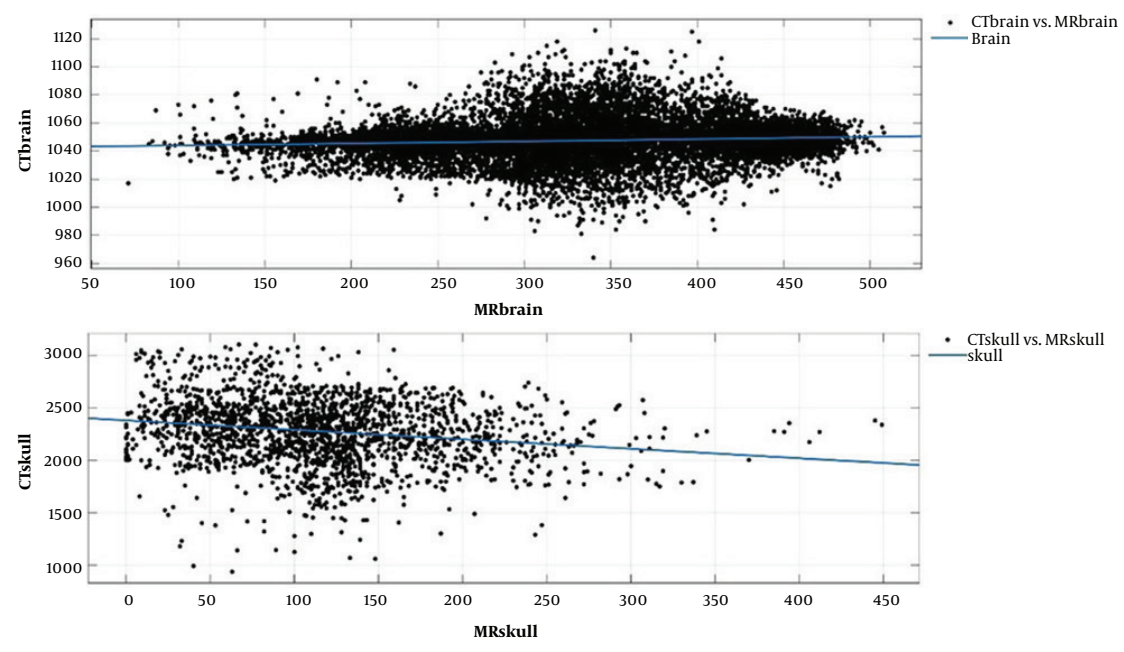

Figure 1. The relationship between MRI intensity and Hounsfield unit (HU) number of voxels within the brain and skull bones

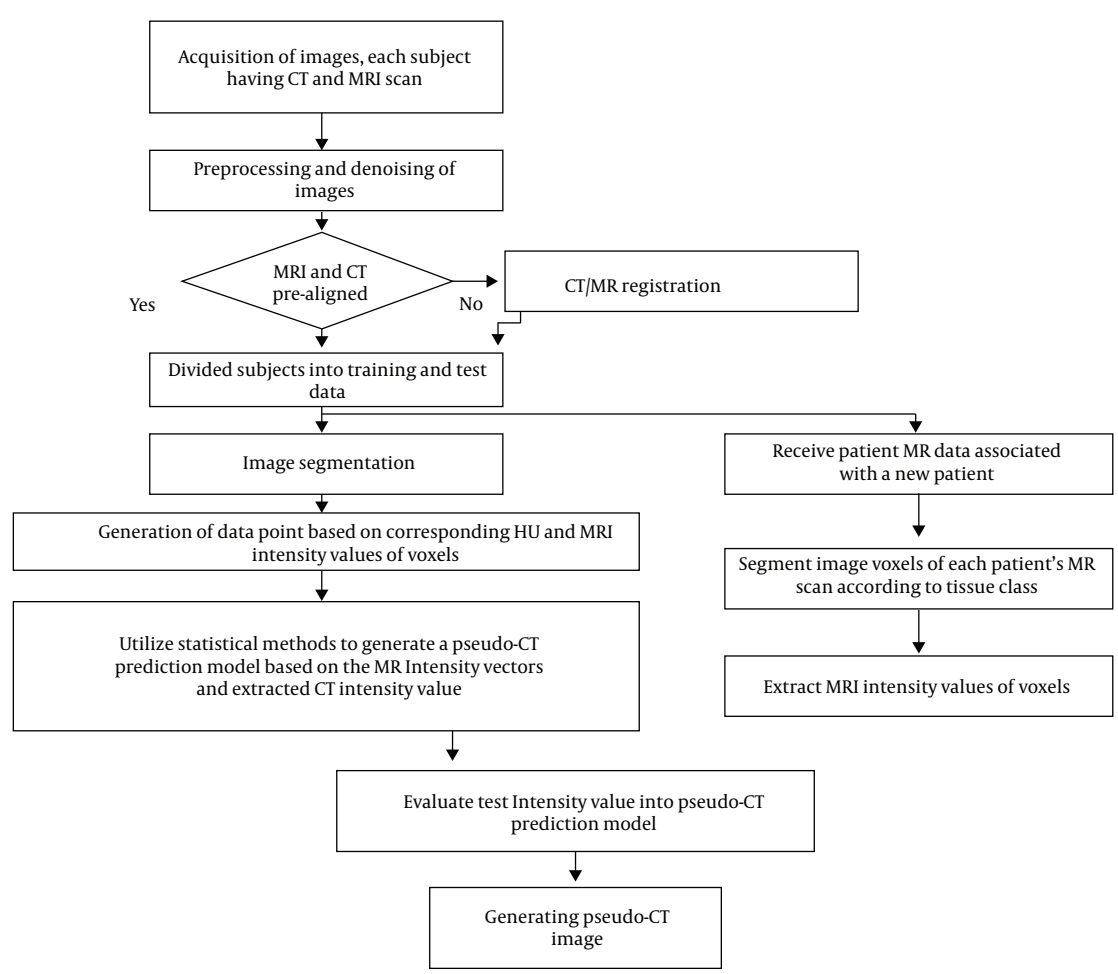

Figure 2. The whole process flowchart

$M A E_{\text {vox }}=\frac{1}{N} \sum_{n=1}^{N}|C T(n)-s C T(n)|$,

$M A E_{\text {vox }}=\frac{1}{N} \sum_{n=1}^{N}(C T(n)-s C T(n))$,
Where, $\mathrm{N}$ is the total number of MR voxels in the different segments, where the CT and pseudo-CT are arranged in a vector of $1^{*} \mathrm{~N}$. They contain the intensity values of the CT and the pseudo-CT in the same location. Table 1 provides the results of the mean absolute error and the mean error for different sections of the head, by applying the polyno- 


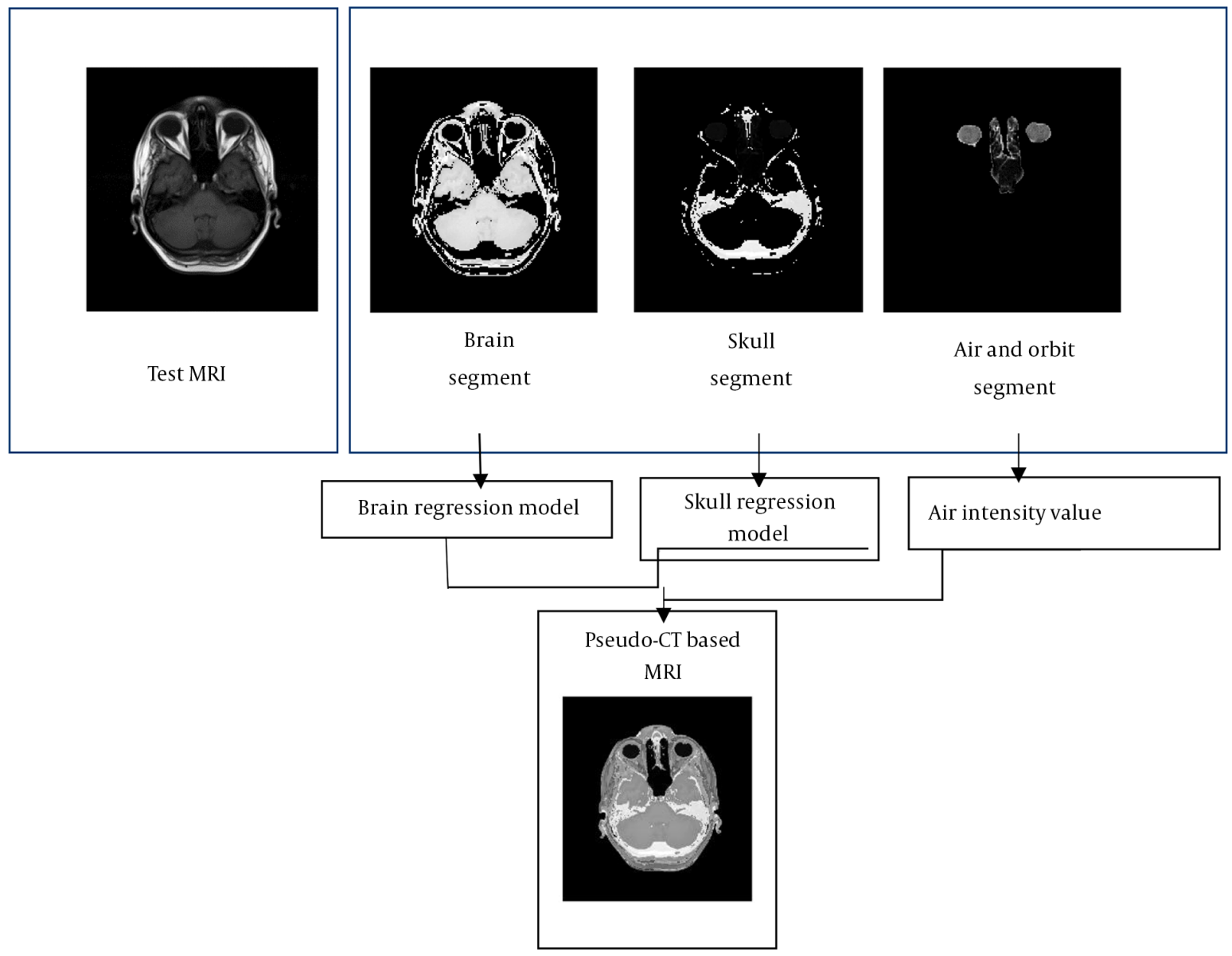

Figure 3. An example of segmentation of images with the aim of creating pseudo-CT using regression models

mial models with the 1st and the 2 nd order.

\section{Discussion}

A relationship has been indicated between the intensity of MR image and CT-based planning (relative electron density information) within the different brain regions with this type of MRI protocol. There is no need for special protocol and multiple MR series to replace CT. The regression relationships between MRI intensity and the relative electron density value from synthetic CT were obtained via the first- and 2nd-order polynomial regression models. An advantage of the proposed method is better distinction of tumor tissue from normal tissue in pseudo-CT images rather than typical CT scan images. It is due to the differences in tumor and normal tissue intensity in MR image and this difference could also be observed after mapping in pseudo-CT image. So the exact tumor delineation can be performed in pseudo-CT as well as MR image. The provided conversion models were validated inside different regions of the head in an independent patient group by choosing the voxels randomly and manually. The results suggested that the 2nd-order model did not generate less error than the first-order model. Because the amount of errors were too close and it could be concluded that by making models more complex, we cannot necessarily expect better results. The correlation values, resulting from the pseudo-CT images and measured CT scans of the patient that were being tested were $0.9682,0.6389$, and 0.7296 in different areas of the brain, skull, air, and sinuses, respectively. It is found that this level of correlation is appropriate in the brain region. The parameter value for the structural similarity index (SSIM) in these images was also equal to 0.8080, 0.9823, and 0.8921 , respectively.

The SSIM can be used for measuring image quality by pseudo-CT image and original CT images as the reference 

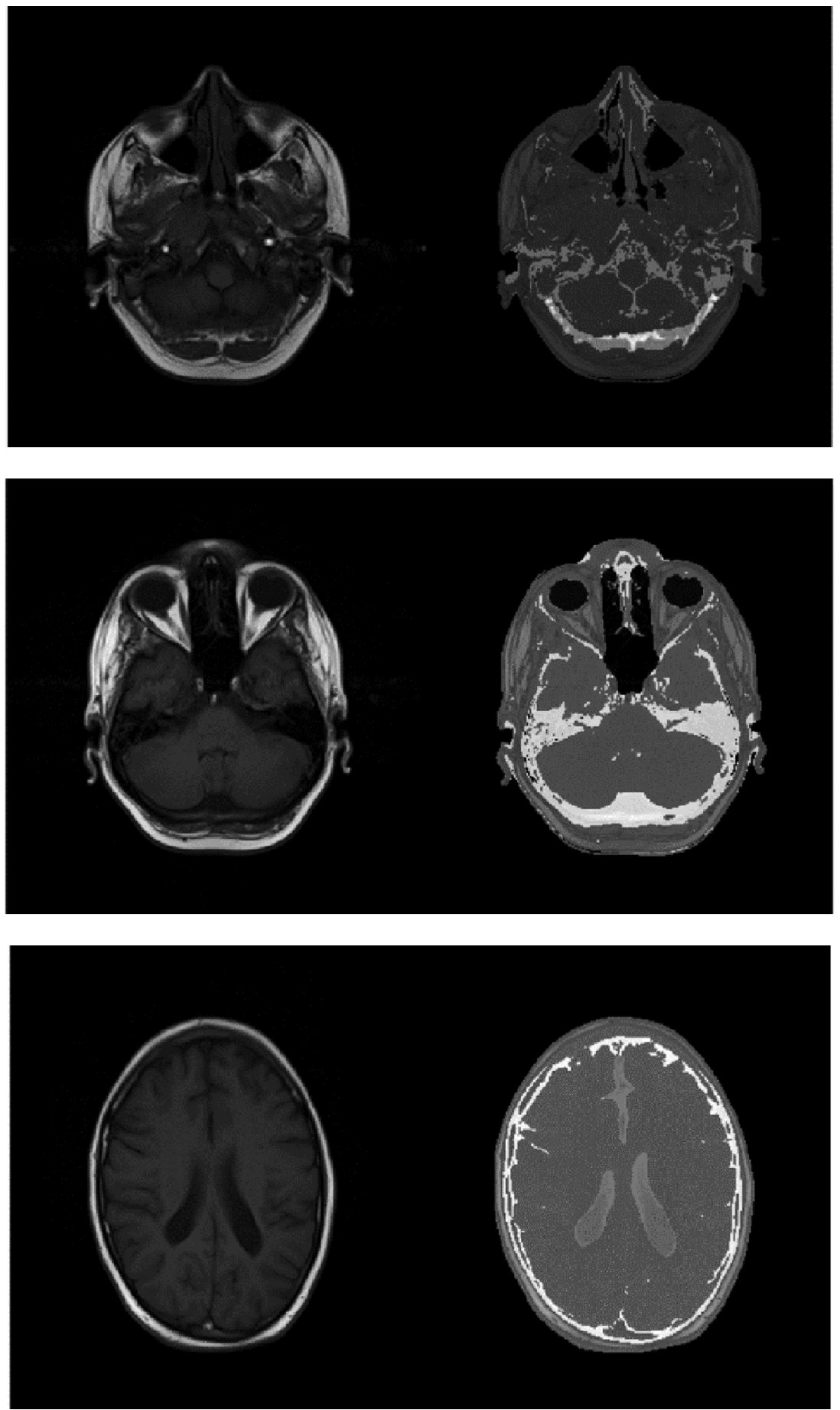

Figure 4. Pseudo-CT generation using regression models for three different slices of the head

image. In the following, the correlation and SSIM value between the whole complete pseudo-CT image and measured images were 0.8567 , and 0.6023 , respectively. In this article, there were less error rates than similar methods in voxel-based articles. In a study conducted by Kapanen and Tenhunen, results are obtained on the pelvic bone area 

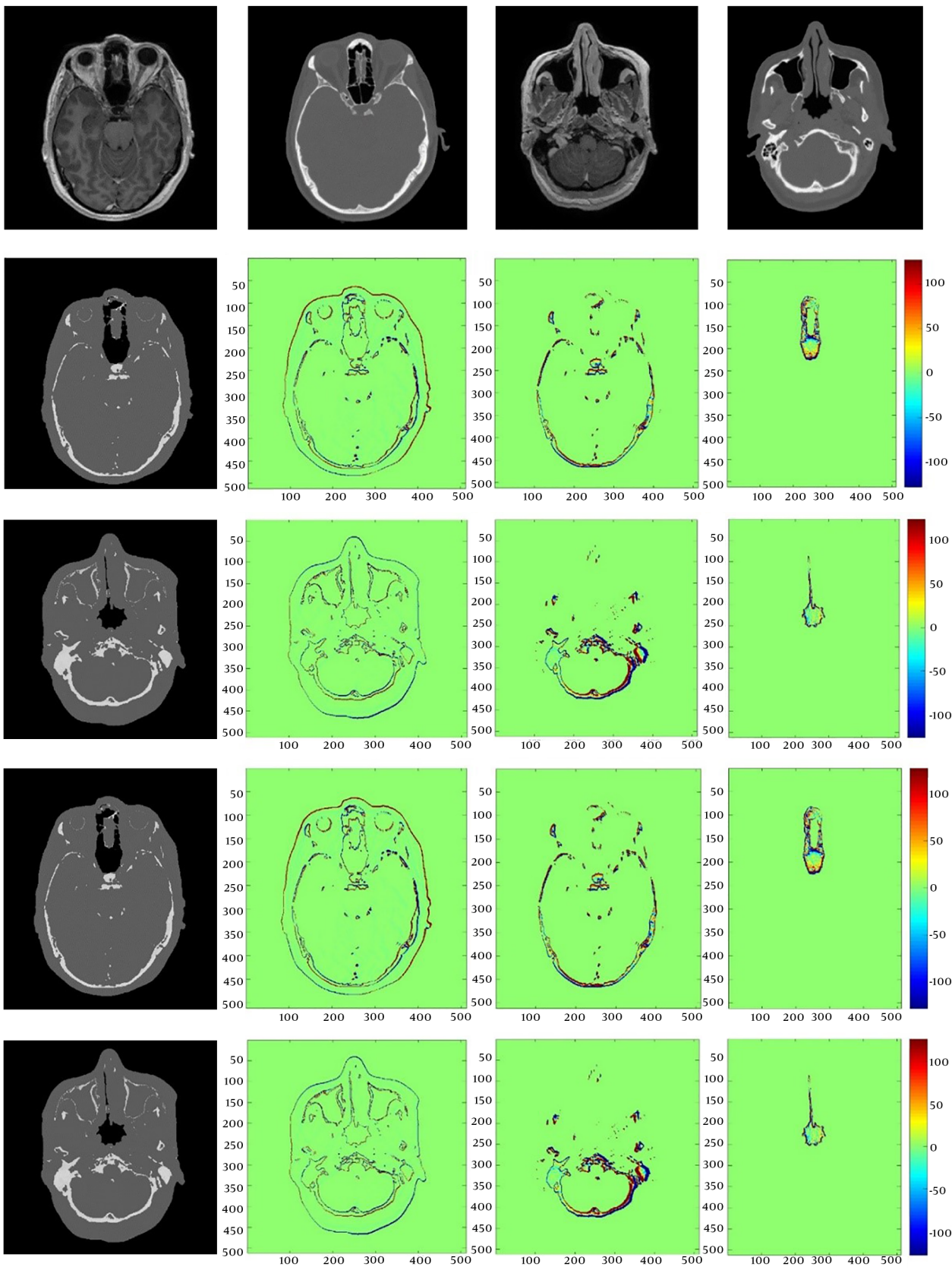

Figure 5. Images in the first row show the original MRI and CT slices. The 2nd and 3rd rows show the pseudo-CT images generated by the 1st-order polynomial model along with their differences between the original and pseudo-CT in the brain, skull, and air region. The 4th and 5th row represent the pseudo-CT images which are generated by the 2nd-order polynomial model along with their differences between original and pseudo-CT in the brain, skull, and air region respectively.

and the MAE of the conversion model was $135 \mathrm{HU}$, while in this article, distinct models have been created on different brain regions with the use of FCM and contouring segmentation (23). The presented model creates appropriate images in terms of graphical observations of the structure of the skull bones and mean square errors. According to the imaging protocol (T1/T2*-weighted), in Kapanen and Tenhunen's study, the results were sensitive to bone segmentation errors (23). Nonetheless, in this study, with the help of convenient segmentation techniques, separation of the skull bone from the air region was appropriate and we were able to separate these areas fairly well and create 

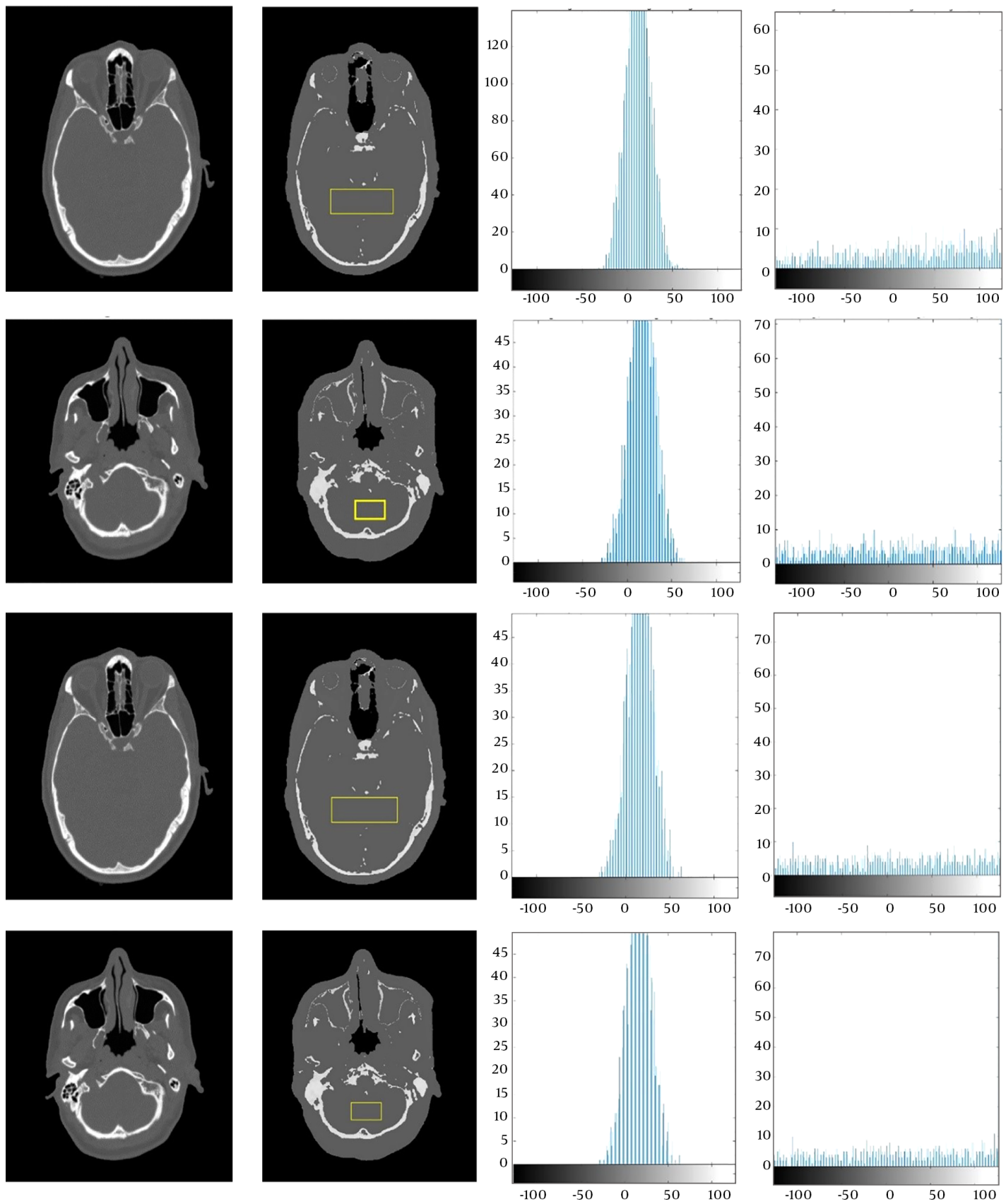

Figure 6. Images in the first column represent the original CT slices. Images in the second column show the pseudo-CT images generated by the two models, and images in the third and fourth columns indicate the differential histograms between the original and pseudo-CT images in the brain and skull region, respectively.

pseudo-CT images with presented regression models. Andreasan et al. used a Gaussian mixture regression (GMR) model with k-means clustering and an EM algorithm to train the model and generate the pseudo-CT by dUTE protocol and add the mDixon images. Nonetheless, these images are not a common protocol in MRI imaging and can limit clinical applications. In addition, the value of the mean absolute prediction error was carried out as $148 \mathrm{HU}$ in their study (42). Other methods such as patch-based regression model and multi-atlas approach based on affine registra- 


\begin{tabular}{|c|c|c|c|c|c|c|c|c|c|c|}
\hline \multirow{2}{*}{ Error } & \multicolumn{3}{|c|}{ Brain: 1st order; skull: 1st order } & \multicolumn{2}{|c|}{ Sub-image 1st model } & \multicolumn{3}{|c|}{ Brain: 2nd order; skull: 2nd order } & \multicolumn{2}{|c|}{ Sub-image 2nd model } \\
\hline & Brain & Skull & Air & Brain & Skull & Brain & Skull & Air & Brain & Skull \\
\hline \multicolumn{11}{|l|}{ Upper slice } \\
\hline ME & 0.7479 & 0.334 & -0.0433 & 7.0088 & -40.944 & 0.7477 & 0.34 & -0.0433 & 11.523 & 10.266 \\
\hline \multicolumn{11}{|l|}{ Middle slice } \\
\hline ME & -0.53 & -0.082 & 0.0145 & 16.759 & -61.276 & -0.53 & -0.060 & 0.0145 & 16.20 & -60.335 \\
\hline MAE & 19.40 & 50.53 & 1.226 & 18.5366 & 119.635 & 19.43 & 50.41 & 1.226 & 18.118 & 119.780 \\
\hline
\end{tabular}

Abbreviations: MAE, mean absolute error; ME, mean error

tions were implemented by Anderson, and 87, and $97 \mathrm{HU}$ mean absolute error (MAE) errors were gained $(30,43)$. The results show that the errors obtained in this study reached the desired level. As a future work, the clinical uncertainty which is related to computing the electron density value by presented conversion models could be clinically tested and become more acceptable.

\section{Footnotes}

Authors' Contributions: All authors contributed equally in developing the original idea and protocol, selecting cases, data acquisition, analyzing data, and preparing the manuscript.

Conflict of Interests: The authors declare that they have no conflicts of interest.

Ethical Approval: This research was approved by Ethical Committee of Shahid Beheshti University of Medical Sciences (number ID: IR.SBMU.MSP.REC.1397.55).

Financial Disclosure: None declared.

Funding/Support: This study was funded and supported by Shahid Beheshti University of Medical Sciences (SBMU).

\section{References}

1. Dowling JA, Sun J, Pichler P, Rivest-Henault D, Ghose S, Richardson H, et al. Automatic substitute computed tomography generation and contouring for magnetic resonance imaging (MRI)-alone external beam radiation therapy from standard MRI sequences. Int J Radiat Oncol Biol Phys. 2015;93(5):1144-53. doi: 10.1016/j.ijrobp.2015.08.045. [PubMed: 26581150].

2. Stanescu T, Hans-Sonke J, Stavrev P, Fallone BG. 3T MR-based treatment planning for radiotherapy of brain lesions. Radiol Oncol. 2006;40(2).

3. Koshy M, Pentaleri M, Paryani S, Anderson C, Cesaretti J, Deshmukh A. The role of MRI in radiation therapy planning. Jacksonville, Florida: FROG MRI Center of North Florida; 2018.

4. Boettger T, Nyholm T, Karlsson M, Nunna C, Celi JC. Radiation therapy planning and simulation with magnetic resonance images. Proc. SPIE 6918, Medical Imaging 2008: Visualization, Image-Guided Procedures, and Modeling, 69181C. 17 March 2008; San Diego, California, United States. 2008.
5. Metcalfe P, Liney GP, Holloway L, Walker A, Barton M, Delaney GP, et al. The potential for an enhanced role for MRI in radiationtherapy treatment planning. Technol Cancer Res Treat. 2013;12(5):42946. doi: 10.7785/tcrt.2012.500342. [PubMed: 23617289]. [PubMed Central: PMC4527434].

6. Rank CM, Hunemohr N, Nagel AM, Rothke MC, Jakel O, Greilich S. MRI-based simulation of treatment plans for ion radiotherapy in the brain region. Radiother Oncol. 2013;109(3):414-8. doi: 10.1016/j.radonc.2013.10.034. [PubMed: 24268939].

7. Liney G, Rai R, Holloway L, Vinod S. A dedicated MRI scanner for radiotherapy planning: Early experiences. Clin Radiat Ther. 2014.

8. Chin AL, Lin A, Anamalayil S, Teo BK. Feasibility and limitations of bulk density assignment in MRI for head and neck IMRT treatment planning. J Appl Clin Med Phys. 2014;15(5):4851. doi: 10.1120/jacmp.v15i5.4851. [PubMed: 25207571]. [PubMed Central: PMC5711084].

9. Wegener D, Zips D, Thorwarth D, Nikolaou K, Othman A, Grosse U, et al. EP-1604: Precision of MRI-CT-image fusion based on goldmarkers for IGRT by using a phantom and clinical data. Radiother Oncol. 2018;127:S863-4. doi: 10.1016/s0167-8140(18)31913-3.

10. Alipour SHM, Houshyari M, Mostaar A. A novel algorithm for PET and MRI fusion based on digital curvelet transform via extracting lesions on both images. Electron Physician. 2017;9(7):4872-9. doi: 10.19082/4872. [PubMed: 28894548]. [PubMed Central: PMC5587006].

11. Mostaar A, Houshyari M, Badieyan S. A novel active contour model for MRI brain segmentation used in radiotherapy treatment planning. Electron Physician. 2016;8(5):2443-51. doi: 10.19082/2443. [PubMed: 27382457]. [PubMed Central: PMC4930267].

12. Stanescu T, Jans HS, Pervez N, Stavrev P, Fallone BG. A study on the magnetic resonance imaging (MRI)-based radiation treatment planning of intracranial lesions. Phys Med Biol. 2008;53(13):3579-93. doi: 10.1088/0031-9155/53/13/013. [PubMed: 18560047].

13. Bakker CJ, Moerland MA, Bhagwandien R, Beersma R. Analysis of machine-dependent and object-induced geometric distortion in 2DFT MR imaging. Magn Reson Imaging. 1992;10(4):597-608. [PubMed: 1501530].

14. Chen Z, Ma CM, Paskalev K, Li J, Yang J, Richardson T, et al. Investigation of MR image distortion for radiotherapy treatment planning of prostate cancer. Phys Med Biol. 2006;51(6):1393-403. doi: 10.1088/00319155/51/6/002. [PubMed: 16510951].

15. Karotki A, Mah K, Meijer G, Meltsner M. Comparison of bulk electron density and voxel-based electron density treatment planning. J Appl Clin Med Phys. 2011;12(4):3522. doi: 10.1120/jacmp.v12i4.3522. [PubMed: 22089006]. [PubMed Central: PMC5718732].

16. Dowling JA, Lambert J, Parker J, Salvado O, Fripp J, Capp A, et al. An atlas-based electron density mapping method for magnetic resonance imaging (MRI)-alone treatment planning and adaptive 
MRI-based prostate radiation therapy. Int J Radiat Oncol Biol Phys. 2012;83(1):e5-11. doi: 10.1016/j.ijrobp.2011.11.056. [PubMed: 22330995].

17. Beavis AW, Gibbs P, Dealey RA, Whitton VJ. Radiotherapy treatment planning of brain tumours using MRI alone. Br J Radiol. 1998;71(845):544-8. doi: 10.1259/bjr.71.845.9691900. [PubMed: 9691900].

18. Greer PB, Dowling JA, Lambert JA, Fripp J, Parker J, Denham JW, et al. A magnetic resonance imaging-based workflow for planning radiation therapy for prostate cancer. Med J Aust. 2011;194(4):S24-7. [PubMed: 21401484].

19. Kapanen M, Collan J, Beule A, Seppala T, Saarilahti K, Tenhunen M. Commissioning of MRI-only based treatment planning procedure for external beam radiotherapy of prostate. Magn Reson Med. 2013;70(1):127-35. doi: 10.1002/mrm.24459. [PubMed: 22886780].

20. Kjer HM. MRI in radiation therapy planning: The use of ultrashort echo time imaging. Technical University of Denmark, Informatics and Mathematical Modelling, Technical University, Denmark; 2012.

21. Jonsson JH, Karlsson MG, Karlsson M, Nyholm T. Treatment planning using MRI data: An analysis of the dose calculation accuracy for different treatment regions. Radiat Oncol. 2010;5:62. doi:10.1186/1748-717X-562. [PubMed: 20591179]. [PubMed Central: PMC2909248].

22. Jonsson JH, Johansson A, Soderstrom K, Asklund T, Nyholm T. Treatment planning of intracranial targets on MRI derived substitute CT data. Radiother Oncol. 2013;108(1):118-22. doi: 10.1016/j.radonc.2013.04.028. [PubMed: 23830190].

23. Kapanen M, Tenhunen M. T1/T2*-weighted MRI provides clinically relevant pseudo-CT density data for the pelvic bones in MRI-only based radiotherapy treatment planning. Acta Oncol. 2013;52(3):612-8. doi: 10.3109/0284186X.2012.692883. [PubMed: 22712634].

24. Korhonen J, Kapanen M, Keyrilainen J, Seppala T, Tuomikoski L, Tenhunen M. Absorbed doses behind bones with MR image-based dose calculations for radiotherapy treatment planning. Med Phys. 2013;40(1):11701. doi: 10.1118/1.4769407. [PubMed: 23298071].

25. Persson E. MRI only radiotherapy using synthetic CT images Dosimetric accuracy and patient positioning strategies in an MRI only workflow [dissertation]. Department of Medical Radiation Physics ,Clinical Science, Lund University; 2015.

26. Sjoberg C, Lundmark M, Granberg C, Johansson S, Ahnesjo A, Montelius A. Clinical evaluation of multi-atlas based segmentation of lymph node regions in head and neck and prostate cancer patients. Radiat Oncol. 2013;8:229. doi: 10.1186/1748-717X-8-229. [PubMed: 24090107]. [PubMed Central: PMC3842681]

27. Rivest-Henault D, Dowson N, Greer PB, Fripp J, Dowling JA. Robust inverse-consistent affine CT-MR registration in MRI-assisted and MRIalone prostate radiation therapy. Med Image Anal. 2015;23(1):56-69. doi:10.1016/j.media.2015.04.014. [PubMed: 25966468].

28. Rivest-Hénault D, Dowson N, Greer P, Dowling J. Inverse-consistent rigid registration of $\mathrm{CT}$ and MR for MR-based planning and adaptive prostate radiation therapy.J Physics Conf Ser. 2014;489:12039. doi: 10.1088/1742-6596/489/1/012039.

29. Burgos N, Cardoso MJ, Guerreiro F, Veiga C, Modat M, McClelland J, et al. Robust CT synthesis for radiotherapy planning: Application to the head and neck region. International Conference on Medical Image Computing and Computer-Assisted Intervention. 2015 Oct 5. Springer; 2015. p. 476-84.

30. Andreasen D. Creating a pseudo-CT from MRI for MRI-only based radiationtherapy planning [dissertation]. Technical University of Denmark; 2013.
31. Jonsson JH, Akhtari MM, Karlsson MG, Johansson A, Asklund T, Nyholm T. Accuracy of inverse treatment planning on substitute CT images derived from MR data for brain lesions. Radiat Oncol. 2015;10:13. doi: 10.1186/s13014-014-0308-1. [PubMed: 25575414]. [PubMed Central: PMC4299127].

32. Zheng W, Kim JP, Kadbi M, Movsas B, Chetty IJ, Glide-Hurst CK. Magnetic resonance-based automatic air segmentation for generation of synthetic computed tomography scans in the head region. Int J Radiat Oncol Biol Phys. 2015;93(3):497-506. doi: 10.1016/j.ijrobp.2015.07.001. [PubMed: 26460991].

33. Korhonen J, Kapanen M, Keyrilainen J, Seppala T, Tenhunen M. A dual model HU conversion from MRI intensity values within and outside of bone segment for MRI-based radiotherapy treatment planning of prostate cancer. Med Phys. 2014;41(1):11704. doi: 10.1118/1.4842575. [PubMed: 24387496].

34. Rank CM, Tremmel C, Hunemohr N, Nagel AM, Jakel O, Greilich S. MRI-based treatment plan simulation and adaptation for ion radiotherapy using a classification-based approach. Radiat Oncol. 2013;8:51. doi: 10.1186/1748-717X-8-51. [PubMed: 23497586]. [PubMed Central: PMC3702461].

35. Whelan B, Kumar S, Dowling J, Begg J, Lambert J, Lim K, et al. Utilising pseudo-CT data for dose calculation and plan optimization in adaptive radiotherapy. Australas Phys Eng Sci Med. 2015;38(4):561-8. doi: 10.1007/s13246-015-0376-z. [PubMed: 26337163].

36. Su KH, Hu L, Stehning C, Helle M, Qian P, Thompson CL, et al. Generation of brain pseudo-CTs using an undersampled, singleacquisition UTE-mDixon pulse sequence and unsupervised clustering. Med Phys. 2015;42(8):4974-86. doi: 10.1118/1.4926756. [PubMed 26233223]. [PubMed Central: PMC5148184].

37. Kuo J, Su K, Hu L, Pereira G, Herrmann K, Muzic R, et al. Feature selection and clustering optimization for pseudo-CT generation in MR based attenuation correction and radiation therapy planning. AAPM Annual Meeting. 2015

38. Johansson A, Karlsson M, Nyholm T. CT substitute derived from MR sequences with ultrashort echo time. Med Phys. 2011;38(5):2708-14. doi: 10.1118/1.3578928. [PubMed: 21776807].

39. Johansson A, Karlsson M, Yu J, Asklund T, Nyholm T. Voxel-wise uncertainty in CT substitute derived from MRI. Med Phys. 2012;39(6):328390. doi: 10.1118/1.4711807. [PubMed: 22755711].

40. Johansson A, Garpebring A, Karlsson M, Asklund T, Nyholm T. Improved quality of computed tomography substitute derived from magnetic resonance (MR) data by incorporation of spatial information-potential application for MR-only radiotherapy and at tenuation correction in positron emission tomography. Acta Oncol. 2013;52(7):1369-73. doi: 10.3109/0284186X.2013.819119. [PubMed 23984810].

41. Bezdek JC, Ehrlich R, Full W. FCM: The fuzzy C-means clustering algorithm. Comput Geosci. 1984;10(2-3):191-203. doi: 10.1016/00983004(84)90020-7.

42. Andreasen D, Andersen JL, Hansen RH, Van Leemput K, Edmund JM The impact of a Dixon sequence in creating a pseudo CT scan from MR images using a Gaussian mixture regression model. Radiother Oncol. 2013;106. S229. doi:10.1016/s0167-8140(15)32899-1.

43. Andreasen D, Van Leemput K, Hansen RH, Andersen JA, Edmund JM. Patch-based generation of a pseudo CT from conventional MRI sequences for MRI-only radiotherapy of the brain. Med Phys 2015;42(4):1596-605. doi: 10.1118/1.4914158. [PubMed: 25832050]. 